\title{
Cambios alimenticios en tres especies de Sphoeroides (Tetraodontiformes: Tetraodontidae) posterior al huracán Isidoro en Bocana de la Carbonera, Sureste del Golfo de México
}

\author{
Sonia Eugenia Palacios-Sánchez \& María Eugenia Vega-Cendejas \\ Laboratorio de Taxonomía y Ecología de Peces, CINVESTAV-IPN, Unidad Mérida, km 6 antigua carretera a Progreso, \\ AP 73 Cordemex. 97310 Mérida, Yucatán, México; soniaepalaciossanchez@gmail.com, \\ maruvega@mda.cinvestav.mx
}

Recibido 17-VIII-2009. Corregido 20-VI-2010. Aceptado 22-VII-2010.

\begin{abstract}
Feeding changes for three Sphoeroides species (Tetraodontiformes: Tetraodontidae) after Isidore hurricane impact in Carbonera Inlet, Southeastern Gulf of Mexico. The coexistence of ecologically similar species may occur because of resources distribution, such as prey and habitat type and segregation time, that minimizes the interspecific competition. The changes brought about by Hurricane Isidore in the distribution of food resources by three coexisting fish species of the family Tetraodontidae (Sphoeroides nephelus, $S$. spengleri and $S$ testudineus), were analyzed at the Carbonera Inlet. Sphoeroides spp. based their food on benthic organisms; principally, they consume mussels (Brachidontes sp.), barnacles (Balanus sp.) and gastropods (Crepidula sp). Before hurricane impact, the three species share the available food resources in different proportions (bivalves, gastropods, barnacles and decapods), according to different strategies that enabled them to coexist and reduce interspecific competition. After the impact, the abundance of available prey decreased and the interespecific competition for food increased, leading to S. testudines and S. nephelus change their trophic spectrum (xiphosurans, amphipods, isopods and detritus) and displacing $S$. splengleri of the inlet. The distribution of food resources was conditioned by the abundance and diversity of prey, as well as the adaptive response of each species. Rev. Biol. Trop. 58 (4): 1223-1235. Epub 2010 December 01.
\end{abstract}

Key words: resource partitioning, feeding habits, coexistence, Sphoeroides spp., Gulf of Mexico.

La presencia de especies del mismo género en un ecosistema, reduce el acceso a los recursos alimenticios requeridos para su sobrevivencia, crecimiento o reproducción. Sin embargo, la coexistencia de las especies con ecología similar puede darse debido a la repartición de recursos, tales como el tipo de presa, hábitat y tiempo de segregación, los cuales minimizan la competencia interespecífica (Pianka 1973, Sala \& Ballesteros 1997, Wootom 1998, Brännäs 2008). Si los recursos son abundantes la competencia es mínima o no sucede por lo que las especies coexistentes pueden exhibir un incremento en la amplitud o sobrelapamiento del nicho, pero si el acceso a los recursos es limitado por cambios en el medio natural, la competencia interespecífica puede intensificarse debido a que algunas especies cambian su perfil de comportamiento y hábitos alimenticios para acceder a los nuevos recursos, mientras otras son segregadas por el hábitat o la dieta (Loureiro-Crippa \& Hahnn 2006, Di Iulio-Ilarri et al. 2008).

Las especies de la familia Tetraodontidae habitan el litoral tropical y subtropical, aunque algunas especies están confinadas en aguas someras sobre fondos arenosos o fangosos (Böhlke \& Chaplin 1993, Randall 1996, SmithVaniz et al. 1999, Mc Eachran \& Fechhelm 2005). Las especies Sphoeroides testudineus y $S$. spengleri, se distribuyen a lo largo de la costa oeste del Atlántico Norte, desde Florida 
hasta Brasil, mientras que $S$. nephelus tiene su principal área de distribución en el Mar Caribe y la parte este del Golfo de México, con poca abundancia en Centroamérica y ausente en las costas de América del Sur (Thayer et al. 1987, Carpenter 2002, Acero \& Polanco 2006, Díaz \& Gómez-López 2003). En algunos sistemas costeros del sureste del Golfo de México, estas tres especies cohabitan y comparten los recursos existentes de forma parcial en diferentes épocas del año (Castro-Aguirre et al. 1999, Vega-Cendejas 2004, Vega-Cendejas \& Hernández de Santillana 2004, Ordóñez-López \& García-Hernández 2005). A pesar de que son similares en sus patrones de conducta, hábitos alimenticios y morfología (cuerpo, boca), se ha observado que existe una repartición de los recursos que facilita su coexistencia, de tal forma que $S$. testudineus por su frecuencia y abundancia es la especie residente, $S$. spengleri es un visitante ocasional y $S$. nephelus utiliza estos sistemas como áreas de protección, alimentación y crianza de juveniles (MallardColmenero et al. 1982). En este sentido, en el presente estudio se analizan los patrones de repartición de recursos alimenticios de estas tres especies de Sphoeroides en la Bocana de la Carbonera (sureste del Golfo de México), antes y después de ser impactada por el huracán Isidoro (septiembre 2002) el cual destruyó su hábitat natural, que paulatinamente se ha ido recuperando.

\section{MATERIALES Y MÉTODOS}

Área de Estudio: La Bocana de la Carbonera se ubica en el sureste del Golfo de México a $21 \mathrm{~km}$ del puerto de altura de Progreso, Yucatán $\left(21^{\circ} 13^{\prime} 41.80^{\prime \prime}-21^{\circ} 14^{\prime} 4.79^{\prime \prime} \mathrm{N}\right.$, $89^{\circ} 53$ '21.66" $\left.-89^{\circ} 54^{\prime} 0.45^{\prime \prime} \mathrm{W}\right)$. Es una entrada de agua de mar que se formó en 1988 al impactar el huracán Gilberto sobre la costa, rompiendo la isla de barrera que separaba el mar de la ciénaga. Con una longitud aproximada de $1 \mathrm{~km}$, era paralela a la costa y limitaba al norte con una franja arenosa, al sur con la duna costera que presentaba en su borde manglares enanos y al este se unía con la ciénaga (Fig. 1A). En septiembre del 2002 el Huracán Isidoro impactó de nuevo la zona, modificando la forma de la Bocana con la desaparición del cordón arenoso que la formaba, quedando la ciénaga en contacto directo con el mar (Fig. 1D). Sin embargo, en los años siguientes la franja de arena se ha ido restableciendo gradualmente recobrando su forma original.

Trabajo de campo: Considerando las dificultades logísticas para realizar muestreos nocturnos así como el comportamiento alimenticio reportado para los tetraodontidos (Targett 1978, Mallard-Colmenero et al. 1982, ClemenceauValdivia 1996), se realizaron ocho muestreos diurnos en la Bocana de la Carbonera (8:00 a.m. y 12:00 p.m.) en octubre de 2001, febrero, mayo, agosto y diciembre de 2002; abril y julio de 2003 y marzo de 2004, que corresponden a los periodos climáticos característicos de la región (nortes, secas y lluvias). Antes del huracán (oct/2001-ago/2002), se establecieron tres sitios de recolecta: uno cerca de la boca que conectaba al mar y dos en la zona interna de la Bocana (Fig. 1A). Posterior al impacto del huracán Isidoro (dic/2002-mar/2004) los sitios de recolecta se modificaron de acuerdo con la nueva morfología del sistema, pero tratando de seguir el mismo patrón de ubicación (Fig. 1D).

Para la recolecta de los peces se utilizó un chinchorro playero de $15 \mathrm{~m}$ de largo por 1.5 $\mathrm{m}$ de ancho, con una abertura de malla de una pulgada. A los ejemplares recolectados se les inyectó formol al $30 \%$ en la cavidad abdominal y se colocaron en bolsas de plástico para su traslado al laboratorio en donde se midieron, pesaron e identificaron utilizando claves taxonómicas especializadas (Fischer 1978, Dickson \& Moore 1998, Castro-Aguirre et al. 1999, McEachran \& Fechhelm 2005).

Análisis Trófico: Debido a que los tetraodontidos presentan un estómago poco desarrollado ya que la mayor parte de la digestión se realiza en el intestino (Breder \& Clarck 1947, Targett 1978), se decidió examinar todo el tracto digestivo (estómago e intestino). Los diversos componentes de la 


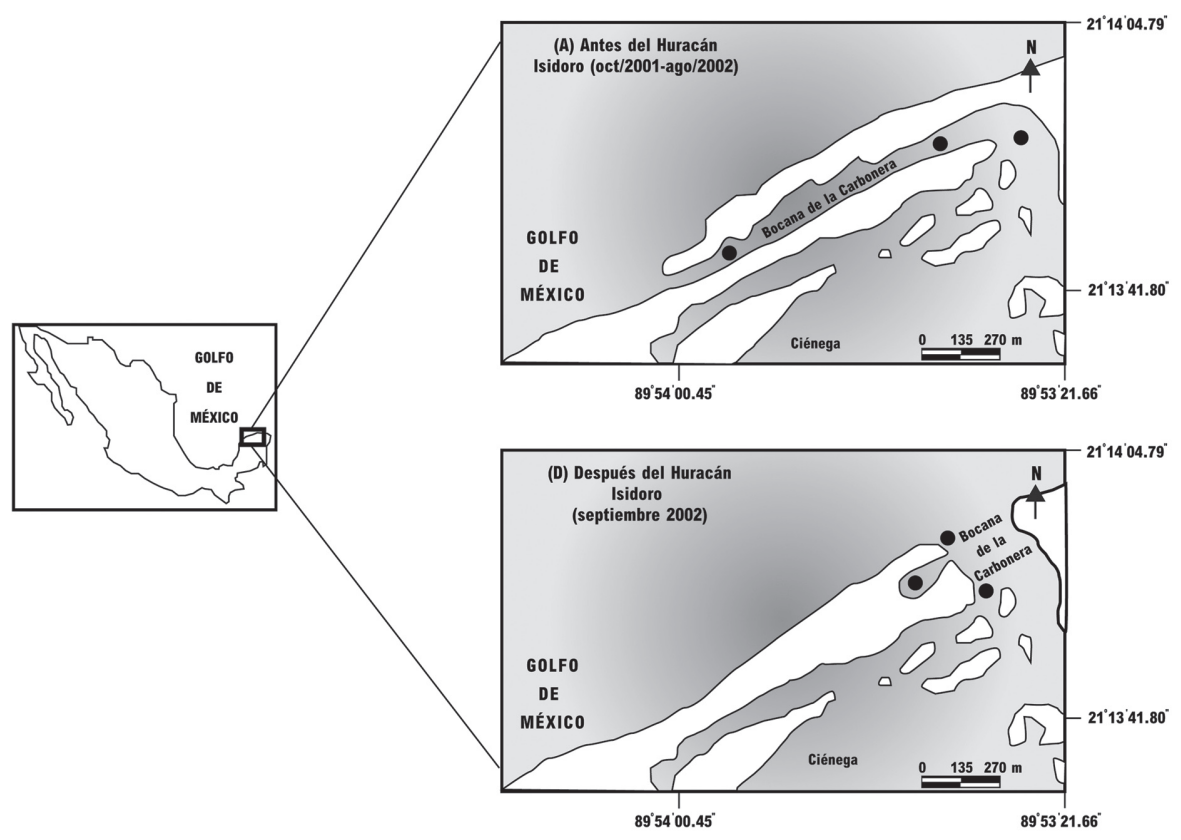

Fig. 1. Área de estudio y estaciones de recolecta antes (A) y después (D) del huracán Isidoro (septiembre 2002) en la Bocana de la Carbonera, Yucatán, Sureste del Golfo de México.

Fig. 1. Study area and sampling sites before (A) and after (D) the impact of the hurricane Isidore (September 2002) on Carbonera Inlet, Yucatan, Southeastern Gulf of Mexico.

dieta se identificaron hasta el nivel taxonómico que permitió la degradación de las presas, se pesaron y clasificaron en categorías taxonómicas. El espectro trófico se determinó a través del método de frecuencia de ocurrencia (FO), el cual establece la proporción de organismos que consumen un determinado artículo alimenticio (Hyslop 1980). Las presas se clasificaron como preferenciales ( $\mathrm{FO}>50 \%)$, secundarias $(\mathrm{FO}>5<50 \%)$ e incidentales $(\mathrm{FO}<5 \%)$ (YáñezArancibia 1975). Para evaluar y comparar la importancia relativa de cada categoría trófica se utilizó el Índice de Importancia Relativa (IRI), en donde el porcentaje de frecuencia de ocurrencia $(\% \mathrm{FO})$ de cada una de las presas es multiplicado por la suma del porcentaje en peso $(\% \mathrm{~W})$ y el porcentaje en número $(\% \mathrm{~N})$ (Cortez 1997).

El grado de utilización de los recursos alimenticios entre las tres especies de Sphoeroides se evaluó a través de la sobreposición de sus dietas (proporción en peso de las presas) mediante un análisis de agrupación utilizando como medida de similitud el índice de Morisita (1959) modificado por Horn (1966), debido a que éste índice es independiente del tamaño de muestra y la diversidad de las presas dentro de una muestra (Wolda 1981).

\section{RESULTADOS}

Artículos alimenticios: Se recolectaron un total de 188 ejemplares (Sphoeroides spp.) con un intervalo de longitud estándar (LE) entre 30 y $203 \mathrm{~mm}$, de los cuales fueron examinados 137 tractos digestivos con material alimenticio: 67 correspondieron a Sphoeroides nephelus (30-79mm LE), 57 a S. testudineus (52-203mm LE) y 13 a $S$. spengleri $(32-45 \mathrm{~mm}$ 
LE). En general, se registraron 91 presas y se clasificaron en 29 categorías taxonómicas siendo los bivalvos y gasterópodos las categorías alimenticias más frecuentes y abundantes, así como las que presentaron la mayor diversidad de presas. Asimismo, las presas más consumidas correspondieron al mejillón Brachidontes sp. (87.59\%), la lapa Balanus sp. (30.65\%) el gasterópodo Crepidula sp. (27.01\%) y el detritus $(27.01 \%)$ (Cuadro 1).
Composición de la dieta: Previo al impacto del huracán Isidoro S. testudineus y S.nephelus presentaron dietas similares al compartir en diferentes proporciones el 50\% de sus artículos alimenticios. Se caracterizaron por tener como presa preferencial al mitílido Brachidontes sp. y por la abundancia de presas secundarias como Balanus sp., Crepidula sp. y decápodos. Por otra parte, S. spengleri presentó como presas preferenciales al mejillón Brachidontes

\section{CUADRO 1}

Composición de dietas de Sphoeroides spp. expresadas como frecuencia de ocurrencia $(F O)$ antes $(A)$ y después $(D)$ del huracán Isidoro en la Bocana de la Carbonera, Sureste del Golfo de México

TABLE 1

Diet composition of Sphoeroides spp. expressed as frequency of occurrence (FO) before $(A)$ and after $(D)$ the impact of hurricane Isidore in Carbonera inlet, Southeastern Gulf of Mexico

\begin{tabular}{|c|c|c|c|c|c|}
\hline \multirow{2}{*}{ Presas } & \multicolumn{3}{|c|}{ (A) } & \multicolumn{2}{|c|}{ (D) } \\
\hline & S. spengleri & S. testudineus & S. nephelus & S. testudineus & S. nephelus \\
\hline No. Estómagos analizados & 13 & 33 & 54 & 24 & 13 \\
\hline Rango de longitud (mm) & $32-45$ & $68-195$ & $30-79$ & $52-203$ & $38-68$ \\
\hline \multirow[t]{2}{*}{ No. Artículos alimenticios } & 18 & 50 & 54 & 39 & 20 \\
\hline & $\% \mathrm{FO}$ & $\% \mathrm{FO}$ & $\% \mathrm{FO}$ & $\% \mathrm{FO}$ & $\% \mathrm{FO}$ \\
\hline \multicolumn{6}{|l|}{ Cyanophiceae } \\
\hline Cianofitas spp. Indet & & 12.12 & & & \\
\hline Oscillatoria spp. & 69.23 & 12.12 & 7.41 & 4.17 & \\
\hline \multicolumn{6}{|l|}{ Bacillarophyceae } \\
\hline Amphora spp. & & & 1.85 & & \\
\hline Cladophora spp. & & 6.06 & & & \\
\hline \multicolumn{6}{|l|}{ Zooplancton } \\
\hline Copépodos Harpacticoideos & & & 1.85 & & \\
\hline Huevos de decápodo & & & 1.85 & & 30.77 \\
\hline Anthozoa Indet. & & 6.06 & & 4.17 & \\
\hline \multicolumn{6}{|l|}{ Foraminiferida } \\
\hline Polystomella spp. & 7.69 & 3.03 & 1.85 & 8.33 & 7.69 \\
\hline \multicolumn{6}{|l|}{ Macrophyta } \\
\hline Pastos marinos spp. indet. & 7.69 & 15.15 & 12.96 & 4.17 & \\
\hline \multicolumn{6}{|l|}{ Algae } \\
\hline Algas spp. indet. & & 6.06 & 9.26 & 8.33 & \\
\hline Rodófitas spp. indet. & & 6.06 & 1.85 & 12.50 & \\
\hline Jania spp. & & & & 4.17 & \\
\hline Algas epífitas spp. indet. & & 15.15 & & 8.33 & \\
\hline \multicolumn{6}{|l|}{ Porifera } \\
\hline Esponjas spp. indet. & & & 1.85 & & \\
\hline \multicolumn{6}{|l|}{ Sipunculida } \\
\hline Sipuncúlidos spp. indet. & & 9.09 & 5.56 & 4.17 & \\
\hline
\end{tabular}


CUADRO 1 (Continuación)

Composición de dietas de Sphoeroides spp. expresadas como frecuencia de ocurrencia (FO) antes (A) y después (D) del huracán Isidoro en la Bocana de la Carbonera, Sureste del Golfo de México

TABLE 1 (Continued)

Diet composition of Sphoeroides spp. expressed as frequency of occurrence (FO) before (A) and after (D) the impact of hurricane Isidore in Carbonera inlet, Southeastern Gulf of Mexico

\begin{tabular}{|c|c|c|c|c|c|}
\hline \multirow{2}{*}{ Presas } & \multicolumn{3}{|c|}{ (A) } & \multicolumn{2}{|c|}{ (D) } \\
\hline & S. spengleri & S. testudineus & S. nephelus & S. testudineus & S. nephelus \\
\hline
\end{tabular}

Ophistobranchia

Opistobranquios spp. indet.

12.12

3.70

Bivalvia

Bivalvos spp. indet.

3.70

Anomalocardia spp.

15.15

4.17

7.69

Anomalocardia auberiana

15.15

Brachodontes spp.

76.92

84.85

Chione cancellata

15.15

Chione spp.

9.09

98.15

62.50

100.00

20.83

15.15

4.17

15.38

Heterodonax bimaculatus

3.03

Macrocallista spp.

3.70

$\left(\frac{10}{20}\right.$

1.85

Mactra spp.

3.03

Musculus spp.

Pandora spp.

Parastarte pustulata

Tellina spp.

Trachycardiun spp.

Transennella spp.

Transennella stimpsoni

Gastropoda

Gasterópodos spp. indet.

23.08

27.27

70.37

29.17

16.67

6.06

1.85

15.38

Alvania spp.

7.69

6.06

4.17

Alvania pelagica

90.9

11.1

Anachis spp.

Batillaria minima

6.06

Bulla spp.

3.03

7.41

Bittium varium

7.69

15.15

7.41

Caecum spp.

9.09

Cerithidea costata

15.15

Cerithium spp.

36.36

5.56

25.00

15.38

Cerithium muscarum

Crepidula spp.

Nerita virginica

Odostomia spp.

4.17

8.33

46.15

Odostome cincta

Olivella spp.

3.03

\subsection{5}

48.15

4.17

Pleuroploca gigantea

Tricolia affinis 
CUADRO 1 (Continuación)

Composición de dietas de Sphoeroides spp. expresadas como frecuencia de ocurrencia (FO) antes (A) y después (D) del huracán Isidoro en la Bocana de la Carbonera, Sureste del Golfo de México

TABLE 1 (Continued)

Diet composition of Sphoeroides spp. expressed as frequency of occurrence (FO) before (A) and after (D) the impact of hurricane Isidore in Carbonera inlet, Southeastern Gulf of Mexico

\begin{tabular}{|c|c|c|c|c|c|}
\hline \multirow{2}{*}{ Presas } & \multicolumn{3}{|c|}{ (A) } & \multicolumn{2}{|c|}{ (D) } \\
\hline & S. spengleri & S. testudineus & S. nephelus & S. testudineus & S. nephelus \\
\hline Tricolia bella & & & 14.81 & & \\
\hline Truncatella spp. & 7.69 & & & & \\
\hline Williamia spp. & & & 1.85 & & \\
\hline \multicolumn{6}{|l|}{ Echiura } \\
\hline Equiuridos spp. indet. & & 12.12 & 5.56 & 4.17 & \\
\hline \multicolumn{6}{|l|}{ Polychaeta } \\
\hline Poliquetos spp. indet. & 61.54 & & 1.85 & 8.33 & 15.38 \\
\hline \multicolumn{6}{|l|}{ Olygochaeta } \\
\hline Oligoquetos spp. indet. & 7.69 & & & 8.33 & \\
\hline \multicolumn{6}{|l|}{ Hirudinea } \\
\hline Hirudíneas spp. indet. & & 3.03 & & & \\
\hline \multicolumn{6}{|l|}{ Xiphosura } \\
\hline Limulus polyphemus & & 6.06 & 1.85 & & 7.69 \\
\hline Huevos de Xiphosura & & & & 41.67 & \\
\hline \multicolumn{6}{|l|}{ Amphipoda } \\
\hline Gamarideos spp. indet. & 15.38 & 9.09 & 14.81 & 12.50 & 100.00 \\
\hline Corophium spp. & & & 7.41 & 4.17 & 100.00 \\
\hline \multicolumn{6}{|l|}{ Isopoda } \\
\hline Isópodos spp. indet & 23.08 & 3.03 & 18.52 & 8.33 & \\
\hline Cassidinea spp. & & & & & 38.46 \\
\hline Cassidinea ovalis & & & 11.11 & & \\
\hline Edotea spp. & & & 7.41 & & \\
\hline \multicolumn{6}{|l|}{ Mysidacea } \\
\hline Misidaceos spp. indet. & & & 1.85 & & \\
\hline \multicolumn{6}{|l|}{ Ostracoda } \\
\hline Ostrácodos spp. indet. & 69.23 & & 3.70 & 12.50 & 15.38 \\
\hline \multicolumn{6}{|l|}{ Tanaidacea } \\
\hline Tanaidaceos spp. indet. & 30.77 & & 5.56 & 8.33 & \\
\hline Leptochelia spp. & 15.38 & & & 4.17 & \\
\hline \multicolumn{6}{|l|}{ Decapoda } \\
\hline Decápodos spp. indet. & 7.69 & 21.21 & 44.44 & 4.17 & 23.08 \\
\hline Farfantepenaeus spp. & & & 1.85 & & \\
\hline \multicolumn{6}{|l|}{ Brachyura } \\
\hline Braquiuros spp. indet. & & 9.09 & 7.41 & 8.33 & \\
\hline Callinectes spp. & & 3.03 & 12.96 & & 7.69 \\
\hline Paguridos spp. indet. & & 18.18 & & 8.33 & \\
\hline Pagurus spp. & & 3.03 & & & \\
\hline Clibanarus vittatus & & 3.03 & & & \\
\hline Emerita talpoida & & 3.03 & & & \\
\hline
\end{tabular}


CUADRO 1 (Continuación)

Composición de dietas de Sphoeroides spp. expresadas como frecuencia de ocurrencia (FO) antes (A) y después (D) del huracán Isidoro en la Bocana de la Carbonera, Sureste del Golfo de México

TABLE 1 (Continued)

Diet composition of Sphoeroides spp. expressed as frequency of occurrence (FO) before (A) and after (D) the impact of hurricane Isidore in Carbonera inlet, Southeastern Gulf of Mexico

\begin{tabular}{|c|c|c|c|c|c|}
\hline \multirow{2}{*}{ Presas } & \multicolumn{3}{|c|}{ (A) } & \multicolumn{2}{|c|}{ (D) } \\
\hline & S. spengleri & S. testudineus & S. nephelus & S. testudineus & S. nephelus \\
\hline \multicolumn{6}{|l|}{ Cirripedia } \\
\hline Lapas spp. indet. & & & 1.85 & & \\
\hline Balanus spp. & & 48.48 & 37.04 & 20.83 & 7.69 \\
\hline \multicolumn{6}{|l|}{ Insecta } \\
\hline Insectos indet. & & & 1.85 & & \\
\hline Hemípteros spp. indet. & & & 1.85 & & \\
\hline \multicolumn{6}{|l|}{ Echinodermata } \\
\hline Equinodermos spp. indet. & & & 5.56 & & \\
\hline \multicolumn{6}{|l|}{ Pisces } \\
\hline Peces spp. indet. & & & & & 7.69 \\
\hline Garmanella pulcra & 7.69 & 3.03 & & & \\
\hline Otras presas & & 3.03 & 40.74 & & \\
\hline Detritus & & 15.15 & 14.81 & 37.50 & 100.00 \\
\hline
\end{tabular}

sp., Cianofitas (Oscillatoria sp.), ostrácodos y poliquetos que constituyeron el $72.46 \%$ de su dieta. La cantidad de artículos alimenticios que S. spengleri incorporó a su alimentación fue menor (18) a las registradas para las otras dos especies (50 y 54).

Posterior al huracán (dic/2002-mar/2004), la ocurrencia y abundancia de las tres especies de Sphoeroides se modificaron, de tal forma que desde diciembre de 2002 no se registraron ejemplares de S. spengleri. En el $2003 S$. nephelus no se recolectó y disminuyó la abundancia de $S$. testudineus; recuperándose ambas poblaciones en el 2004 (Fig. 2). Con respecto a su alimentación, la diversidad trófica para las dos especies (S. testudineus y $S$. nephelus) posterior al huracán fue menor, aunque el mitílido Brachidontes sp. siguió siendo la presa preferencial de mayor abundancia y el detritus la segunda en importancia. Además de éste artículo alimenticio $S$. nephelus incluyó en su dieta como presas preferenciales al detritus y anfípodos (gamarideos y Corophium sp.), mientras que $S$. testudineus presentó como principales presas secundarias huevos de Xiphosura sp., gasterópodos y detritus. Ambas especies incorporaron nuevos artículos alimenticios a sus dietas, como al isópodo Cassidinea sp. y al gasterópodo Cerithium sp. que en conjunto constituyeron el $5.91 \%$ de la dieta de $S$. nephelus. Asimismo S. testudineus incorporó a su alimentación los huevos de Xiphosura sp., al gasterópodo Pleuropoca gigantea, oligoquetos y poliquetos que representaron el $17.71 \%$ de su dieta. Aunque estas dos especies compartieron 14 artículos alimenticios, sus hábitos tróficos difieren debido a que $S$. testudineus incluyó 39 presas en su alimentación en contraste con $S$. nephelus cuya dieta estuvo compuesta por 20 presas (Cuadro 1).

Categorías tróficas y repartición de recursos alimenticios: De acuerdo con el índice de importancia relativa (IRI), las tres especies de Sphoeroides basaron su alimentación en organismos bentónicos con una 


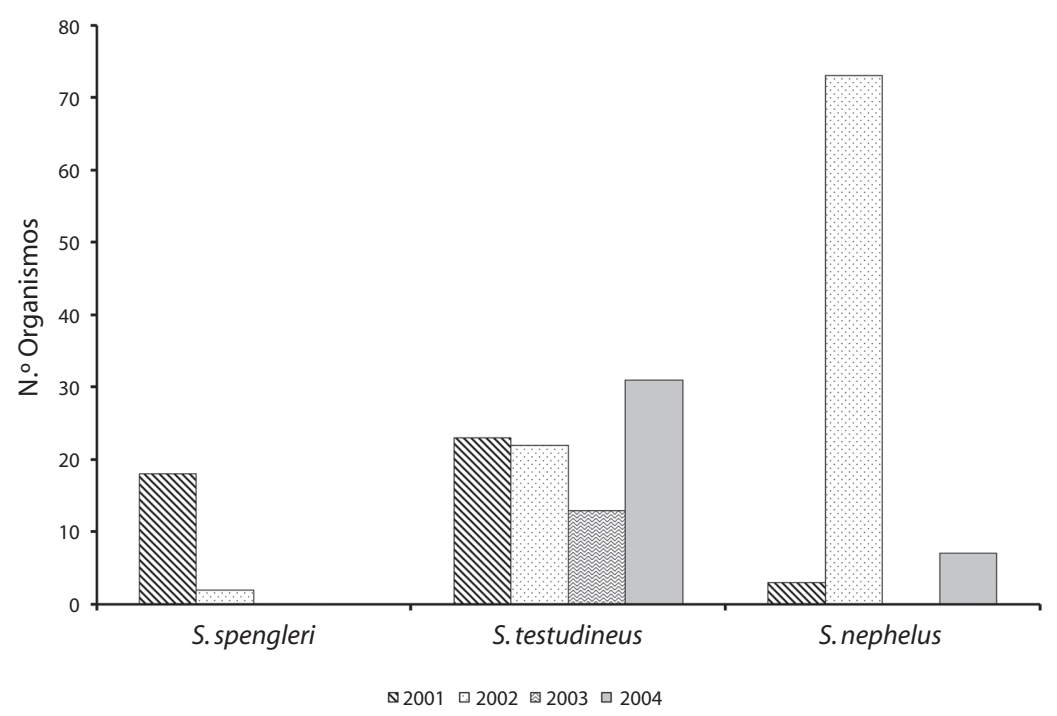

Fig. 2. Número total de Sphoeroides spp. recolectados entre 2001-2004 en la Bocana de la Carbonera, Sureste del Golfo de México.

Fig. 2. Total number of Sphoeroides spp. collected from 2001 to 2004 in Carbonera Inlet, Southeastern Gulf of Mexico.

marcada preferencia hacia los bivalvos. Previo al impacto del huracán, $S$. nephelus y $S$. testudineus presentaron afinidad en los grupos tróficos que integraron sus dietas, así que los bivalvos, gasterópodos, cirrípedos y decápodos fueron los principales recursos alimenticios. En contraste $S$. spengleri utilizó además de los bivalvos otros grupos tróficos disponibles como los poliquetos, ostrácodos, cianofitas y tanaidaceos (Fig. 3). Después que el huracán Isidoro impactó la Bocana de la Carbonera modificando su hábitat, los Sphoeroides spp. cambiaron sus preferencias alimenticias. El $S$. testudineus incorporó en su dieta a los poliquetos y oligoquetos e incrementó el consumo de detritus, braquiuros y límulos, disminuyendo su consumo de bivalvos, gasterópodos cirrípedos y decápodos. El S. nephelus incrementó el consumo de anfípodos, isópodos, ostrácodos, poliquetos, zooplancton y detritus, reduciendo a su vez el de bivalvos, gasterópodos, cirrípedos y decápodos (Fig. 3). En ambas especies algunas categorías tróficas como los opistobranquios y el fitoplancton ya no fueron consumidas después del evento de huracán.
Interacción trófica entre las especies: De acuerdo con el diagrama de similitud derivado del índice de Morisita y a un nivel de corte de 0.5 se formaron dos grupos (Fig. 4), donde se observó que las dietas de $S$. nephelus y $S$. testudineus cambiaron posteriormente al impacto del huracán, utilizando de forma oportuna los recursos que se encontraban disponibles y desplazando de la Bocana a S. spengleri. El grupo uno se dividió en dos subgrupos, en el subgrupo (a) S. testudineus después del huracán presentó una dieta similar (0.55) a la del subgrupo (b) sin embargo la inclusión de bivalvos, gasterópodos y cirrípedos en su dieta fue menor, por lo que integró otras presas a su alimentación tales como los límulos, braquiuros, algas y detritus. El subgrupo (b) estuvo conformado por S. nephelus y S. testudineus, cuyas dietas antes del evento de huracán fueron similares (0.70) compartiendo 19 artículos alimenticios entre los que destacaron los bivalvos, gasterópodos, cirrípedos, cianofitas y detritus. En el grupo dos, la dieta que presentó $S$. spengleri antes del huracán fue similar (0.72) a la que exhibió $S$. nephelus 


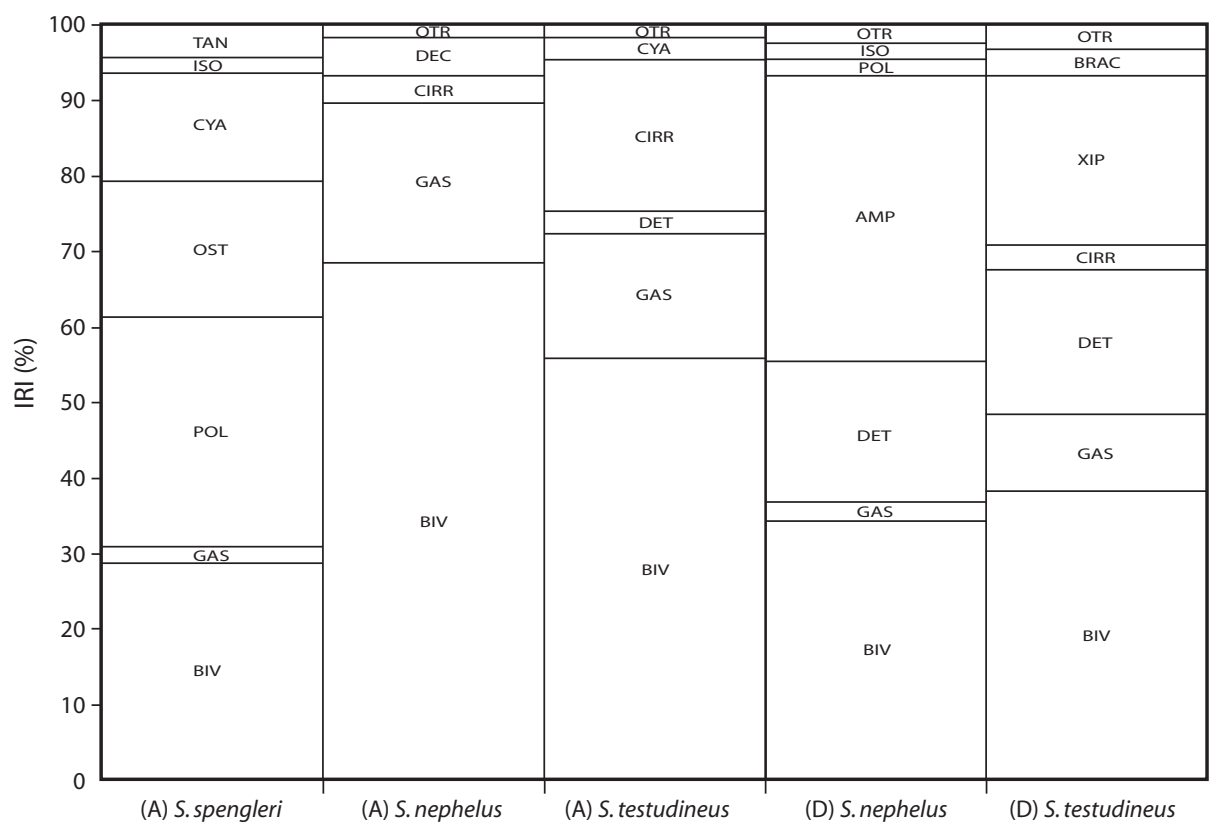

Fig. 3. Preferencias alimenticias de Sphoeroides spp. antes (A) y después (D) del huracán Isidoro (septiembre 2002) en la Bocana de la Carbonera, Sureste del Golfo de México. Los tamaños de clase representan la importancia relativa (IRI) de las principales categorías tróficas (AMP=anfípodos; BIV=bivalvos; BRAC=branquiuros; $\mathbf{C I R R = c i r r i ́ p e d o s ; ~}$ CYA=cianofitas; DEC=decápodos; DET=detritus; GAS=gasterópodos; ISO=isópodos; OST=ostrácodos; OTR=otras presas: $\mathbf{P O L}=$ poliquetos; $\mathbf{T A N}=$ tanaidáceos; $\mathbf{X I P}=$ Xiphosura).

Fig. 3. Feeding preference of Sphoeroides spp. before (A) and after (D) hurricane Isidore (September 2002) in the Bocana of the Carbonera, Southeastern Gulf of Mexico. Every class size represents the relative importance (\%IRI) of the principal trophic categories (AMP=amphipods; $\mathbf{B I V = b i v a l v e s ; ~} \mathbf{B R A C}=$ brachyurans; $\mathbf{C I R R}=$ barnacles; $\mathbf{C Y A}=$ cyanophythes; DEC=decapods; DET=detritus; GAS=gastropods; ISO=isopods; OST=ostracods; OTR=others preys; $\mathbf{P O L}=$ polychaetes; TAN=tanaids; $\mathbf{X I P}=$ xiphosurans).

después del evento meteorológico debido a que ambas especies utilizaron, aunque en diferentes tiempos, los mismos recursos alimenticios (poliquetos, bivalvos, detritus, anfípodos, ostrácodos, gasterópodos y decápodos).

\section{DISCUSIÓN}

Sphoeroides nephelus, S. spengleri y $S$. testudineus presentaron un amplio espectro trófico (91 presas) basando su alimentación en organismos bentónicos, por lo que se comportaron como consumidores de segundo orden. Estas especies exhibieron diferentes patrones de comportamiento en la repartición de los recursos alimenticios antes y después del huracán, lo cual estuvo relacionado con la disponibilidad de presas, confirmando que la repartición de los recursos alimenticios es una respuesta adaptativa a la competencia interespecífica por el alimento o la escasez del mismo (Brännäs 2008).

Los mecanismos que determinan la coexistencia de las especies están relacionados con sus hábitos alimenticios, zonas de alimentación, abundancia de las presas y forma de las estructuras para la alimentación (Targett 1978). En este estudio se determinó que la repartición de los recursos de Sphoeroides spp. previo al impacto se realizó en cuatro patrones: 1) Brachidontes sp. fue la principal presa consumida y se integró en diferentes proporciones a sus 


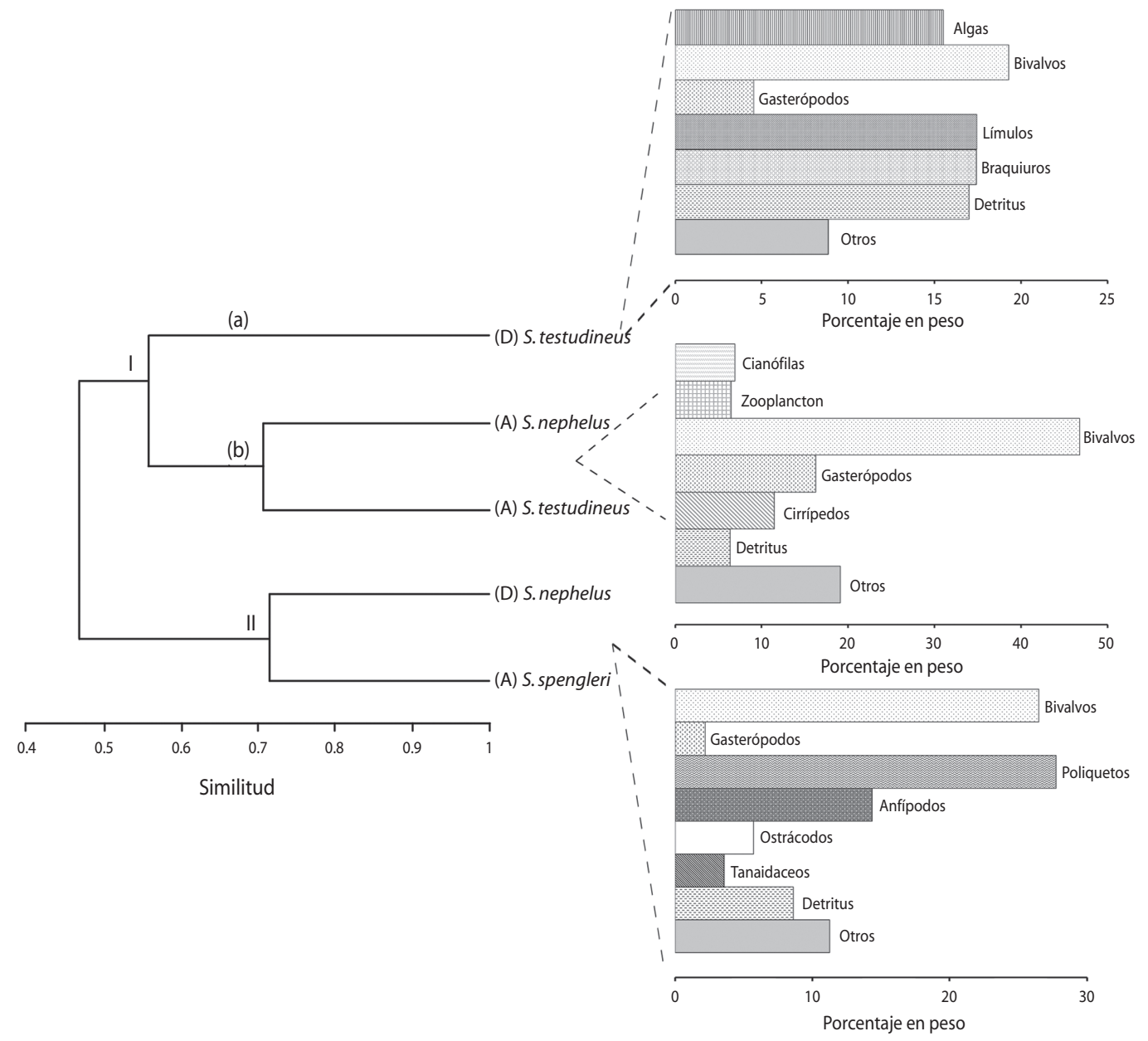

Fig. 4. Dendrograma de similitud trófica de S. nephelus, S. spengleri y S. testudineus antes (A) y después (D) del huracán Isidoro (septiembre 2002) en la Bocana de la Carbonera, Sureste del Golfo de México. Porcentaje en peso de los componentes de la dieta por grupo y subgrupo trófico.

Fig. 4. Trophic similarity cluster for S. nephelus, S. spengleri and S. testudineus before (A) and after (D) the impact of the hurricane Isidore (September 2002) in the Carbonera Inlet, Southeastern Gulf of Mexico. It is showed the percentage by weight of diet composition for each trophic group and subgroup.

dietas, 2) se presentó una selección y exclusión de las presas de una misma categoría trófica (bivalvos y gasterópodos principalmente), 3) se realizó un uso diferenciado de las presas; es decir, el artículo alimenticio más importante para una especie no lo fue para las otras dos y 4) repartición de las zonas de alimentación en relación con la ocurrencia, abundancia y talla de las especies. Este comportamiento fue similar a lo reportado para estas especies por Targett (1978) y Mallard-Colmenero et al. (1982). Sin embargo, en el presente estudio se observó que cuando la disponibilidad de los recursos cambió debido al impacto del huracán, la competencia interespecífica se incrementó provocando que $S$. testudineus y $S$. nephelus cambiaran su espectro trófico desplazando a $S$. spengleri de la Bocana. 
La competencia interespecífica antes del huracán fue mínima, permitiendo la coexistencia de las tres especies que inclusive sobrelaparon parcialmente sus dietas y zonas de alimentación. De tal forma que $S$. testudineus y $S$. nephelus presentaron dietas similares (bivalvos, gasterópodos, cirrípedos y decápodos) pero en diferentes zonas de alimentación. La primera por ser más abundante y tener un mayor rango de tallas (52-203mm LE) se distribuyó en toda la Bocana, mientras que $S$. nephelus, cuyos ejemplares fueron de menor talla y abundancia (30-79mm LE), estuvieron restringidos a las zonas cercanas al manglar, áreas que compartieron con S. spengleri, cuyo espectro trófico fue diferente al de las otras dos especies (cianofitas, bivalvos, poliquetos, ostrácodos y tanaidaceos).

La composición de la dieta de $S$. spengleri fue similar a las reportadas en otros estudios (Randall 1967, 1996, Mallard-Colmenero et al. 1982, Vega et al. 1997), que indican que los anfípodos, isópodos, poliquetos y moluscos son sus principales artículos alimenticios y confirmando que las diferencias observadas en el presente estudio respecto a las dietas de las otras dos especies de Sphoeroides no son un efecto del tamaño de muestra (16 organismos) ó del intervalo de talla analizado (32-45 mm LE).

No obstante lo anterior, se ha observado que Sphoeroides spp. cambia sus hábitos alimenticios con su desarrollo ontogénico (Targett 1978, Clemenceau-Valdivia 1996, Guevara et al. 2007), ya que se vuelven más selectivos con el incremento de talla como respuesta a sus requerimientos fisiológicos y nutricionales (Wooton 1998). Sin embargo, también se ha observado que las presas preferenciales se conservan a través del tiempo cambiando sólo su proporción en la dieta (Targett 1978, Clemenceau-Valdivia 1996). En este sentido y de acuerdo con los intervalos de tallas registrados para las tres especies de Sphoeroides en la Bocana de la Carbonera, aparentemente estas especies sobrelaparon sus dietas pero en realidad la competencia por el alimento previo al impacto del huracán fue mínima debido a que la diversidad de las presas fue elevada favoreciendo la coexistencia de las especies, condición que se modifico posterior al evento.

Las perturbaciones del hábitat provocadas por huracán Isidoro en la costa, disminuyó la abundancia y densidad de los organismos bentónicos (Ardisson \& Castillo-Fernández 2004), por lo que estas presas potenciales para los peces escasearon como se observó en las dietas de $S$. testudineus y $S$. nephelus que integraron al detritus como la tercera categoría trófica en importancia, desplazando a los gasterópodos y cirrípedos de su alimentación. Ante la escasez del alimento $S$. testudineus y $S$. nephelus presentaron un comportamiento oportunista además de una gran flexibilidad trófica que les permitió ajustar sus actividades de forrajeo y permanecer en el sistema a diferencia de $S$. spengleri, que fue excluido del mismo al sobrelapar su dieta (bivalvos, poliquetos, isópodos, ostrácodos) y área de alimentación con $S$. nephelus. Los resultados confirman que $S$. testudineus es una especie exitosa capaz de competir y desplazar a las especies que son ecológicamente cercanas en áreas donde co-ocurren (Shipp 1974, Targett 1978), tal es el caso con S. nephelus, al restringir su alimentación y forzarla a competir por espacio y alimento con $S$. splengleri que fue poco abundante y de tallas muy pequeñas (32$45 \mathrm{~mm} \mathrm{LE}$ ).

Por otra parte, se ha observado que los peces al localizar un ambiente estable hidrológicamente y rico en recursos, lo explotan invirtiendo un menor gasto de energía en su búsqueda y elección de alimento (Granados 1996), lo que podría explicar el uso de Brachidontes sp. como la principal presa de las tres especies de Sphoeroides, ya que este mejillón es común y abundante en sistemas salobres y suele formar colonias masivas sobre los fondos arenosos o fangosos, así como entre las raíces del manglar o las rocas (Morris 1975, Rehder 1981, Escalante et al. 1987). Otra estrategia que utilizaron estas especies para incrementar su eficiencia en el forrajeo y minimizar la competencia interespecífica fue el presentar una especialización o preferencia por algunas presas en particular de bivalvos y gasterópodos, reafirmando que 
la repartición de los recursos alimenticios es resultado de las interacciones competitivas de las especies que los inducen a desarrollar diferentes estrategias para hacer un uso óptimo de los recursos disponibles en el ecosistema.

\section{AGRADECIMIENTOS}

Agradecemos a la Comisión Nacional de Biodiversidad, México (CONABIO) por el financiamiento otorgado (Proyecto Y027) y al Consejo Nacional de Ciencia y Tecnología, México (CONACyT) por la beca conferida durante la investigación doctoral del primer autor. Se agradece de manera especial a Mirella Hernández de Santillana por su valiosa colaboración en el campo y en la identificación de los contenidos estomacales, así como Alex Acosta, Walter Canto y Víctor Castillo por su ayuda en la recolecta de muestras. Un agradecimiento especial a Luis Rene Capurro Filograsso por la revisión y valiosos comentarios sobre el manuscrito.

\section{RESUMEN}

Se analizan los cambios producidos por el huracán Isidoro en la repartición de los recursos alimenticios de tres especies de peces de la familia Tetraodontidae (Sphoeroides nephelus, S. spengleri y S testudineus) que cohabitan en la Bocana de la Carbonera (sureste del Golfo de México). Los Sphoeroides spp. basaron su alimentación en los organismos bentónicos, sobresaliendo por su consumo el mejillón (Brachidontes sp.), la lapa (Balanus sp.) y gasterópodos (Crepidula sp.). Previo al impacto del huracán, las tres especies utilizaron de forma diferencial los recursos alimenticios disponibles (bivalvos, gasterópodos, cirrípedos y decápodos) recurriendo a diferentes estrategias que les permitieron minimizar la competencia interespecífica y coexistir. Posterior al impacto, la disponibilidad de las presas disminuyó y la competencia interespecífica por el alimento se incrementó provocando que $S$. testudineus y $S$. nephelus cambiaran su espectro trófico (xiphosuros, anfípodos, isópodos y detritus) desplazando a $S$. spengleri de la bocana. La repartición de los recursos alimenticios estuvo condicionada por la abundancia y diversidad de las presas así como la respuesta adaptativa de cada especie.

Palabras clave: repartición de recursos, hábitos alimenticios, coexistencia, Sphoeroides spp., Golfo de México.

\section{REFERENCIAS}

Acero, A. \& A. Polanco. 2006. Peces del orden Tetraodontiformes de Colombia. Biota Colombiana. 7: 155-164.

Ardisson P.L. \& D. Castillo-Fernández. 2004. Diversidad bentónica del ambiente intermareal e infralitoral somero de Progreso, Yucatán. CONABIO/CINVESTAV-IPN, Unidad Mérida, Yucatán, México.

Böhlke, J.E. \& C.C. Chaplin. 1993. Fishes of the Bahamas and Adjacent Tropical Waters. University of Texas, Austin, EEUU.

Brännäs, E. 2008. Temporal resources partitioning varies with individual competitive ability: a test with Artic charr Salvelinus alpinus visiting a feeding site from a refuge. J. Fish Biol. 73: 524-535.

Breder, C.M. \& E. Clark. 1947. A contribution to the visceral anatomy, development and relationships of the Plectognathi. Bull. Am. Mus. Nat. Hist. 88: 287-320.

Carpenter, K.E. 2002. The living marine resources of the Western Central Atlantic FAO. Species Identification Guide for Fishery Purposes. Amer. Soc. Ichthyol. Herp. Spec. Publ., Rome.

Castro-Aguirre, J.L., H. Espinosa-Pérez \& J.J. SchmitterSoto. 1999. Ictiofauna estuarino-lagunar y vicaria de México. Limusa, Ciudad de México, México.

Clemenceau-Valdivia, F. 1996. Aspectos de la biología de Sphoeroides testudineus y $S$. nephelus, en la laguna de Tamiahua, Veracruz, México. Tesis de Licenciatura, Universidad Nacional Autónoma de México, México D.F., México.

Cortez, E. 1997. A critical review of methods of studying fish feeding based on analysis of stomach contents: Application to elasmobranches fishes. Can. J. Fish. Aquat. Sci. 54: 726-738.

Díaz, J.M. \& D.I. Gómez-López. 2003. Cambios históricos en la distribución y abundancia de praderas de pastos marinos en la bahía de Cartagena y áreas aledañas (Colombia). Bol. Invest. Mar. Cost. 32: 57-74.

Dickson, H.H. \& R.H. Moore. 1998. Fishes of the Gulf of Mexico. Texas, Louisiana and adjacent waters. Texas A\&M University, Texas, EEUU.

Di Iulio-Ilarri, M., A.T. de Souza, P.R. de Medeiros, R.G. Grempel \& I.M. de Lucena Rosa. 2008. Effects of tourist visitation and supplementary feeding on fish assemblage composition on a tropical ref. in the Southwestern Atlantic. Neotrop. Ichthyol. 6: 651-656.

Escalante, M., D. Leon \& E. Serviere. 1987. Moluscos asociados a macroalgas en la Laguna de Bojorquez 
(Sistema Lagunar Nichupte), Cancún, Quintana Roo. III Reunión Nacional de Malacología, Universidad Autónoma de Nuevo León, México.

Fischer, W. 1978. FAO Species identification sheets for fisheries purposes. Western Central Atlantic. FAO, Roma, Italia.

Granados, L.C. 1996. Ecología de Peces. Universidad de Sevilla, Sevilla, España.

Guevara, E., A.J. Sánchez, C. Rosas, M. Mascaró \& R. Brito. 2007. Asociación trófica de peces distribuidos en vegetación acuática sumergida en la Laguna de Términos, sur del Golfo de México. Uciencia 23: 151-166.

Horn, H.S. 1966. Measurement of overlap in comparative ecological studies. Am. Nat. 100: 419-424.

Hyslop, E.J. 1980. Stomach contents analysis: a review of methods and their application. J. Fish. Biol. 17: 411-429.

Loureiro-Crippa, V.E. \& N.S. Hahnn. 2006. Use of food resources by the fish fauna of a small reservoir (rio Jordão, Brazil) before and shortly after its filling. Neotrop. Ichthyol. 4: 357-362.

Mallard-Colmenero, L., A. Yáñez-Arancibia \& F. Amezcua-Linares. 1982. Taxonomía, biología y ecología de los tetraodontidos de la Laguna de Términos, sur del Golfo de México (Pises: Tetraodontidae). An. Inst. Cienc. del Mar Limnol. Univ. Nal. Auton. México. 9: $161-212$.

McEachran, J.D. \& J.D. Fechhelm. 2005. Fishes of the Gulf of Mexico. Vol. 2, Scorpaeniformes to Tetraodontiformes. University of Texas, Texas, EEUU.

Morris, P.A. 1975. A field guide to shells of the Atlantic and Gulf coasts and West Indies, EEUU. Houghton Mifflin. Boston, Massachusetts, EEUU.

Morisita, M. 1959. Measuring interspecific association and similarity between communities. Univ. Ser. Ed. (Biol.) 3: 65-80

Ordóñez-López U. \& V.D. García-Hernández. 2005. Ictiofauna juvenil asociada a

Thalassia testudinum en Laguna Yalahau, Quintana Roo. Hidrobiológica 15: 195-204.

Pianka, E.R. 1973. The structure of lizard communities. Annu. Rev. Ecol. Syst. 21: 213-220.
Randall, J.E. 1967. Food habits of reef fishes of the West Indies. Stud. Trop. Oceanogr. 5: 665-847.

Randall, J.E. 1996. Caribbean reef fishes. T.F.H., EEUU.

Sala, E. \& E. Ballesteros. 1997. Partitioning of space and food resources by three fish of the genus Diplo$d u s$ /Sparidae) in a Mediterranean rocky infralitoral ecosystem. Mar. Ecol. Prog. Ser. 152: 273-283.

Rehder, H.A. 1981. Field Guide to North American Seashells. Chanticleer, Nueva York, EEUU.

Shipp, R.L. 1974. The pufferfishes (tetraodontidae) of the Atlantic Ocean. Publ. Gulf. Coast Res. Lab. Mus. 4: 1-163.

Smith-Vaniz, W.F., Collette, B.B. \& Luckhurst, B.E. 1999. Fishes of Bermuda: History, zoogeography, annotated checklist, and identification keys. American Society of Ichthyologists and Herpetologists, EEUU.

Targett, T.E. 1978. Food resources partitioning by the Pufferfishes Sphoeroides spengleri and S. testudineus from Biscayne Bay, Florida. Mar. Biol. 49: 83-91.

Thayer G.W., D.R. Colby \& W.F. Hettler, Jr. 1987. Utilization of the red mangrove prop root habitat by fishes in south Florida. Mar. Ecol. Prog. Ser. 35: 25-38.

Vega-Cendejas, M.E. 2004. Ictiofauna de la Reserva de la Biosfera Celestún, Yucatán: una contribución al conocimiento de su biodiversidad. An. Inst. Biol. Univ. Nal. Autón. México, Serie Zoología 75: 193 206.

Vega-Cendejas, M.E. \& M.J. Hernández de S. 2004. Fish community structure and dynamics in a coastal hypersaline lagoon: Rio Lagartos, Yucatan, Mexico. Estuar. Coast. Shelf Sci. 60: 285-299.

Vega-Cendejas, M.E., M. Hernández de Santillana \& G. De la Cruz-Agüero. 1997. Los peces de la reserva de Celestún. Cinvestav-U. Mérida/Pronatura, Mérida, Yucatán, México.

Wolda, H. 1981. Similarity indices, samples size and diversity. Oecologia 50: 296-302.

Wootton, R.J. 1998. Ecology of Teleost. Chapman \& Hall, Londres, Inglaterra.

Yáñez-Arancibia, A. 1975. Estudios de peces en las lagunas costeras: Nota Científica. An. Inst. Cienc. del Mar y Limnol. Univ. Nal. Autón. México 2: 53-60. 